06,11

\title{
Фазовый переход сегнетоэлектрика нитрата калия в нанопористой матрице
}

\author{
(C) В.М. Егоров ${ }^{1}$, Ю.Ф. Марков ${ }^{1}$, Е.М. Рогинский ${ }^{1}$, Е.В. Стукова ${ }^{2}$ \\ ${ }^{1}$ Физико-технический институт им. А.Ф. Иофрфе РАН, \\ Санкт-Петербург, Россия \\ ${ }^{2}$ Амурский государственный университет, \\ Благовещенск, Россия \\ E-mail: victor_egorov1@inbox.ru
}

Поступила в Редакцию 30 марта 2021 г.

В окончательной редакции 30 марта 2021 г.

Принята к публикации 30 марта 2021 г.

\begin{abstract}
Калориметрическим методом изучены нанокомпозиционные материалы, представляющие собой силикатные нанопористые матрицы, заполненные $\mathrm{KNO}_{3}$. Обнаруженные аномалии теплоемкости, связанные с сегнетоэлектрическими фазовыми переходами, изучены в рамках теории размытых фазовых переходов. Экстраполяция полученных результатов подтверждает высказанное ранее предположение о том, что существует минимальный размер пор в нанокомпозите, при котором $\mathrm{KNO}_{3}$ при комнатной температуре будет находиться полностью в сегнетоэлектрическом состоянии.
\end{abstract}

Ключевые слова: фазовые переходы, сегнетоэлектрик, нитрат калия, теплоемкость.

DOI: 10.21883/FTT.2021.08.51162.069

В последнее время большое внимание уделяется изучению нанокомпозиционных материалов, представляющих собой нанопористые матрицы, заполненные различными веществами. Связано это с тем, что характеристики таких композитов могут не аддитивно изменяться в сравнении со свойствами объемных материалов. Происходит это вследствие размерных эффектов, взаимодействия вводимого в поры материала со стенками пор, взаимодействия между наноразмерными частицами вводимого материала. Изучение сегнетоэлектрических композитных материалов на основе нитрата калия находится на начальном этапе, хотя исследованию сегнетоэлектрических свойств объемного $\mathrm{KNO}_{3}$ посвящено большое количество работ. Особое место среди таких композитов занимают композиты на основе наноразмерных матриц с введенными в поры сегнетоэлектрическими частицами. Такие гетероструктуры на основе упорядоченных наноразмерных матриц, заполненных сегнетоэлектриком, могут использоваться при изготовлении элементов памяти долговременных носителей информации, для микроскопических источников питания, пироприемников, высокоемкостных конденсаторов и датчиков для определения влажности $[1,2]$.

В настоящей работе приводятся результаты калориметрического исследования аномалий теплоемкости композитных материалов, связанные с сегнетоэлектрическими фазовыми переходами. Методом исследования являлась дифференциальная сканирующая калориметрия (ДСК). Тепловые свойства образцов исследовались на калориметре DSC „Perkin-Elmer“ в атмосфере азота при нагревании и охлаждении. Температурная шкала калибровалась по точкам плавления льда $(273.1 \mathrm{~K})$ и индия
(429.7 K), а шкала теплового потока - по теплоемкости лейкосапфира. Измерения проводились в области температур $300-450 \mathrm{~K}$.

Образцы для исследования приготовлялись из нанокомпозитов на основе $\mathrm{KNO}_{3}$, внедренного в матрицы MCM (MCM - Mobil Composition of Matter). Исходным являлся образец объемного $\mathrm{KNO}_{3}$ (образец № 1). Материал типа МСМ является хорошо изученным представителем семейства мезопористых материалов на основе диоксида кремния [3]. Структура материала МСМ представляет собой каркас в форме пчелиных сот, диаметр которых $R$ в нашей работе составлял $3.5-3.7 \mathrm{~nm}$ (образец № 2); 2.8-3.0 nm (образец № 3) и 1.9-2.1 nm (образец № 4). Целью работы является получение калориметрических данных о поведении соответствующих максимумов теплоемкости, об изменении области существования сегнетофазы в нанокомпозиционных материалах и изменении условий ее возникновения.

Как известно, в кристаллах $\mathrm{KNO}_{3}$ существует три полиморфные фазы $[4,5]$, взаимные переходы между которыми сопровождаются эндотермическими и экзотермическими эффектами, соответственно при нагревании и охлаждении. Все эти фазовые превращения прослеживаются на наших образцах на кривых ДСК в виде пиков теплоемкости, полученных при нагревании и охлаждении. На рис. 1 представлены для примера исходные кривые ДСК, полученные при нагревании и охлаждении образцов № 1 и 3 .

Так, $\alpha$-фаза (обычно обозначаемая как фаза II), имеющая при комнатной температуре и атмосферном давлении ромбическую структуру, при нагревании перехо- 

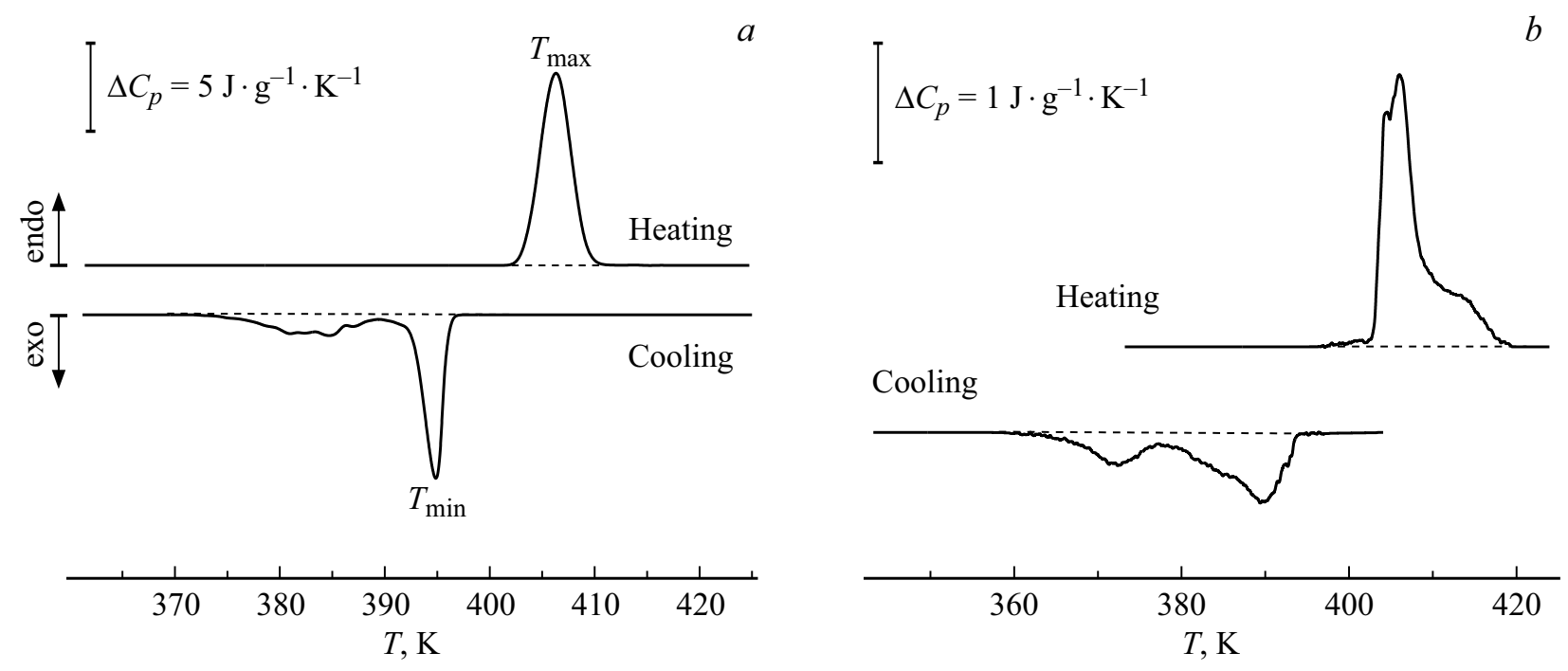

Рис. 1. Исходные кривые ДСК при нагревании и охлаждении образцов № 1 (a) и № 3 (b).

дит в $\beta$-фазу, обычно обозначаемую как фаза I. Этот переход проявляется на кривой ДСК образца № 1 в виде симметричного $\Lambda$-образного пика с температурой максимума $T_{\max }$, а для образца № 3 этот переход происходит практически при той же температуре, но форма эндотермического пика обнаруживает более сложную форму.

$\beta$-фаза имеет тригональную кальцитоподобную структуру. При охлаждении наблюдаются следующие фазовые превращения: сначала $\beta$-фаза переходит в сегнетоэлектрическую $\gamma$-фазу (фаза III). При дальнейшем охлаждении $\gamma$-фаза превращается в исходную перед нагреванием $\alpha$-фазу. Этим переходам на кривых ДСК соответствуют экзотермические пики в виде дублета, отдельные составляющие которого соответствуют последовательному фазовому превращению III $\rightarrow$ I $\rightarrow$ II.

Известно, что температурный диапазон существования сегнетофазы в $\mathrm{KNO}_{3}$ зависит от тепловой предыстории и скорости охлаждения [6] и может составлять температурный интервал $\sim 20 \mathrm{~K}$. В нашем случае при скорости сканирования $v=5 \mathrm{~K} / \mathrm{min}$ температурный интервал $T_{\max }-T_{\min }$ составляет $\sim 10 \mathrm{~K}$. Однако, этот температурный интервал может быть обусловлен как физической причиной, отмеченной выше, так и методическим фактором, поскольку при использовании метода ДСК возникает смещение пиков по температуре. Это смещение связано с наличием термосопротивления испытываемого образца в калориметрической ячейке, которое существенно зависит от скорости сканирования [7]. В работе [8] показано, что эта методическая погрешность может быть устранена следующим образом: по экспериментальным данным при вариации скоростей сканирования $V$ (нагревания или охлаждения) строится зависимость $T_{\max , \min }=f\left(V^{1 / 2}\right)$, которая при отсутствии каких- либо структурных трансформаций должна быть линейной. Экстраполяция этой линейной зависимости

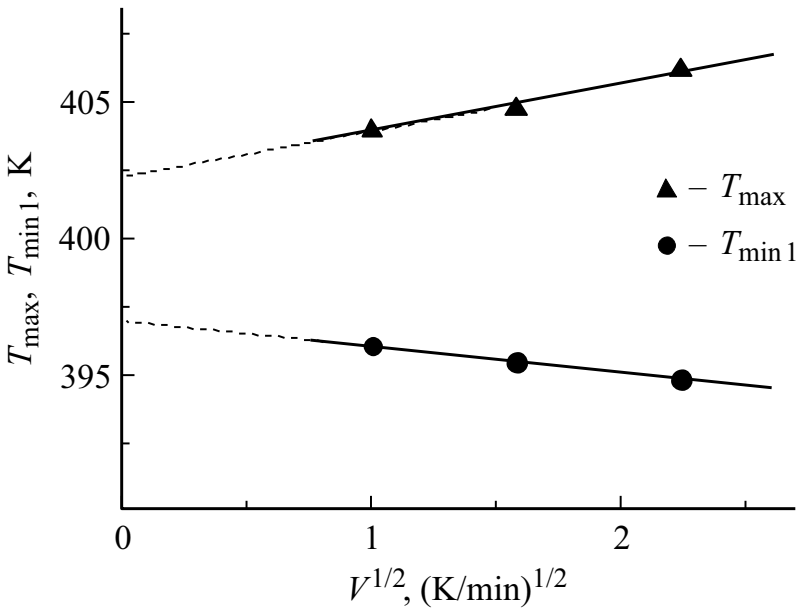

Рис. 2. Зависимость температур $T_{\max }$ и $T_{\min }$ от скорости сканирования для образца № 1.

к $V \rightarrow 0$ позволяет получить истинные, методически неискаженные значения температуры перехода и температурного гистерезиса. В настоящей работе были получены подобные зависимости в циклах нагревание охлаждение для образца № 1 (рис. 2) и определены экстраполированные (истинные) значения температур фазового перехода. Величина температурного гистерезиса составила $\Delta T=T_{\max }-T_{\min }=5.3 \pm 0.2 \mathrm{~K}$.

Полученную зависимость $T_{\max , \min }=f\left(V^{1 / 2}\right)$ для образца № 1 использовали как градуировочную зависимость для других образцов, что существенно упрощает проведение трудоемких ДСК экспериментов для всей серии образцов. Отметим, что в отличие от температурных, экспериментальные данные по энтальпии $(\Delta H)$ и энтропии $(\Delta S)$ в методе ДСК не зависят от скорости сканирования по температуре и определяются по соотношениям $\Delta H=\int C_{p}(T) d T$ и $\Delta S=\int C_{p}(T) d(\ln T)$. 

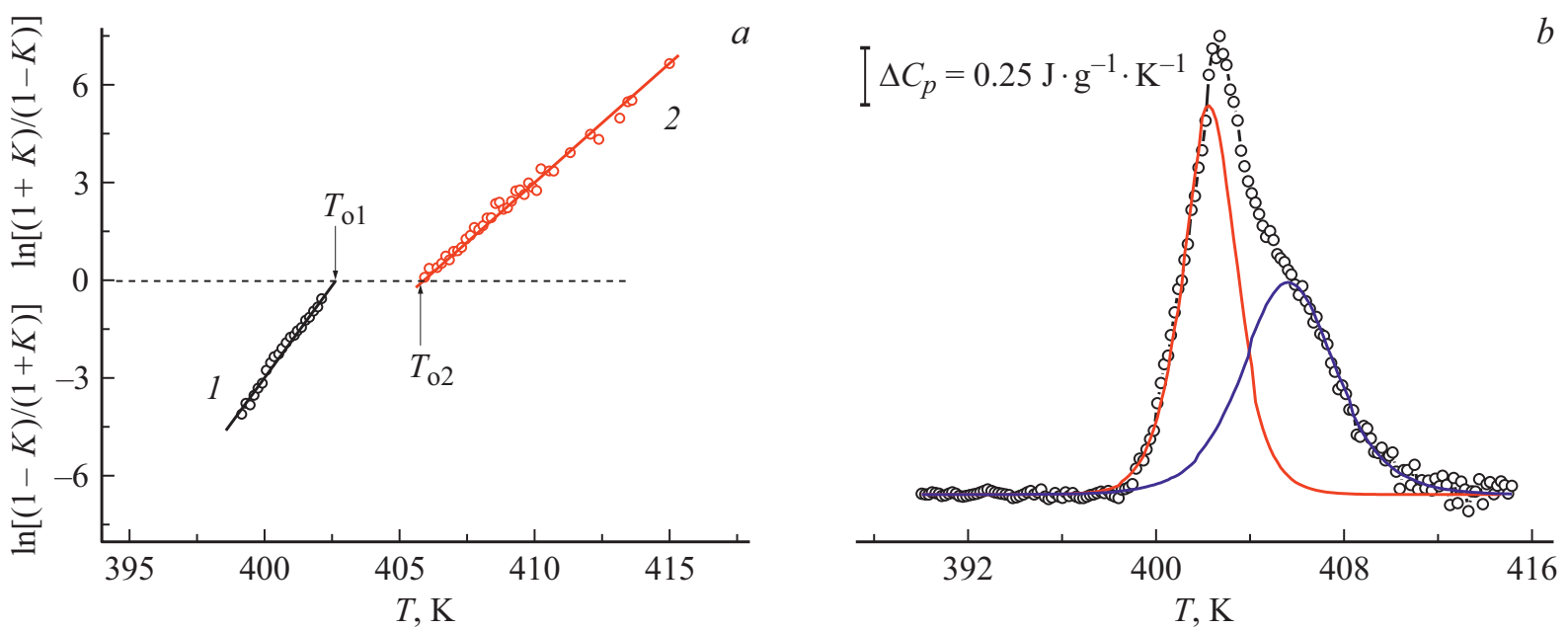

Рис. 3. Разложение несимметричного пика теплоемкости твердофазного перехода на два симметричных пика $\Lambda$-образной формы для образца № 4. (a) - зависимости, рассчитанные по соотношениям (2) и (3), кружки - экспериментальные данные, линии результат расчета. $(b)$ - эндотермические пики теплоемкости, кружки - экспериментальные данные, тонкие линии - результат расчета по зависимости (1).

Суммарные значения энтальпии $\left(\Delta H_{\Sigma}\right)$ для всех исследованных образцов представлены в таблице.

Наличие гистерезиса позволяет на основании данных ДСК отнести этот переход к твердофазному переходу первого рода, а конечный температурный интервал и симметричная $\Lambda$-образная форма пика теплоемкости для образца № 1 позволяют классифицировать его как размытый фазовый переход первого рода $[9,10]$. Форма эндотермического пика для образца № 3 указывает на сложную структуру перехода и представляет интерес для подробного анализа.

Анализ формы пиков теплоемкости проводился на основе теории самосогласованного поля применительно к $\Lambda$-образным размытым переходам первого рода. В размытых переходах изменение фазового состояния происходит в пределах определенного температурного интервала. Образование зародышей-доменов новой фазы требует изменения температуры на малую величину, которая определяется энергией, необходимой для возникновения таких областей. Это и приводит к размытию перехода по температуре. Например, для сегнетоэлектрических материалов было показано, что элементарные объемы превращения сопоставимы с объемом так называемой области Кенцига $\left(\sim 10^{-18}-10^{-17} \mathrm{~cm}^{3}\right)$ и по своему масштабу находятся на мезоскопическом уровне [11].

Температурная зависимость теплоемкости при размытом фазовом переходе имеет следующий вид:

$$
\begin{aligned}
\Delta C_{p}(T)= & 4 \Delta C_{m} \cdot \exp \left[B\left(T-T_{\mathrm{o}}\right) / T_{\mathrm{o}}\right] \\
& \times\left[1+\exp \left[B\left(T-T_{\mathrm{o}}\right) / T_{\mathrm{o}}\right]\right]^{-2},
\end{aligned}
$$

где $T_{\mathrm{o}}$ - температура фазового перехода; $\Delta C_{m}-$ максимальное значение теплоемкости при $T=T_{\mathrm{o}}$; $B$ - атермический параметр [12]. Вводя обозначения $P(T)=\Delta C_{p}(T) / \Delta C_{m}$ и $K=[1-P(T)]^{1 / 2}$, уравнение $(1)$ можно представить в виде линейных зависимостей от температуры

$$
\ln [(1-K) /(1+K)]=B\left(T-T_{\mathrm{o}}\right) / T_{\mathrm{o}}
$$

для высокотемпературного плеча пика, и

$$
\ln [(1+K) /(1-K)]=B\left(T-T_{\mathrm{o}}\right) / T_{\mathrm{o}}
$$

для низкотемпературного плеча. Согласно соотношениям (2) и (3) наклон этих линейных зависимостей определяется параметром $B$. В случае, если форма пика теплоемкости несимметрична или не описывается соотношением (1), это сразу же проявится на линейных зависимостях $\ln [f(K)]=f(T)$. Наличие асимметрии пика, обусловленное наложением двух симметричных пиков, приведет к смещению друг относительно друга по температурной шкале прямолинейных зависимостей на величину, равную разнице между температурами максимумов этих двух пиков. В этом случае есть все основания провести разложение сложного пика на два составляющих симметричных пика.

На рис. 3, $a$ для примера представлены зависимости, рассчитанные по соотношениям (2) и (3), применительно к восходящему (кривая 1) и нисходящему (кривая 2) участкам пика для образца № 4. Видно, что в этих координатах зависимости представляют собой прямые линии. По наклону этих зависимостей, в соответствии с соотношениями (2) и (3), были определены параметры $B$, а по точке пересечения с осью абсцисс $-T_{\mathrm{o} 1}$ и $T_{\mathrm{o} 2}$. Это дает возможность воспользоваться соотношением (1) для построения двух симметричных $\Lambda$-образных пиков (рис. $3, b$ ). Амплитуды этих пиков, т.е. величины $\Delta C_{m 1}$ и $\Delta C_{m 2}$, определялись из условия равенства энтальпии экспериментально полученного пика сумме энтальпий двух расчетных симметричных пиков $\left(\Delta H_{\Sigma}=q_{\mathrm{o} 1}+q_{\mathrm{o} 2}\right)$. 
Параметры, использованные для расчета пиков теплоемкости по соотношению (1)

\begin{tabular}{|c|c|c|c|c|c|c|c|}
\hline \multirow{2}{*}{$\begin{array}{c}\text { № } \\
\text { образца }\end{array}$} & $T_{\mathrm{o} 1}$ & $T_{\mathrm{o} 2}$ & $B$ & $\Delta C_{m}$ & $q_{\mathrm{o} 1}$ & $q_{\mathrm{o} 2}$ & $\Delta H_{\Sigma}$ \\
\hline & \multicolumn{2}{|c|}{ K } & & $\mathrm{J} / \mathrm{gK}$ & $\mathrm{J} / \mathrm{g}$ & $\mathrm{J} / \mathrm{g}$ & $\mathrm{J} / \mathrm{g}$ \\
\hline \multirow{2}{*}{1} & 404.5 & & 1000 & 2.2 & 4.1 & & \multirow[t]{2}{*}{45.1} \\
\hline & & 406.3 & 500 & 12.0 & & 41.0 & \\
\hline \multirow{2}{*}{2} & 403.5 & & 1000 & 3.0 & 4.8 & & \multirow[t]{2}{*}{21.0} \\
\hline & & 409.4 & 200 & 2.0 & & 16.2 & \\
\hline \multirow{2}{*}{3} & 404.2 & & 1200 & 1.2 & 1.6 & & \multirow[t]{2}{*}{8.8} \\
\hline & & 406.1 & 500 & 2.3 & & 7.2 & \\
\hline \multirow[b]{2}{*}{4} & 403.0 & & 500 & 1.5 & 4.4 & & \multirow[t]{2}{*}{8.5} \\
\hline & & 406.4 & 300 & 0.8 & & 4.1 & \\
\hline
\end{tabular}

Параметры расчета составляющих пиков для всех образцов приведены в таблице.

На рис. 4 показаны экспериментальные и рассчитанные по соотношению (1) зависимости теплоемкости от температуры для образцов исходного поликристаллического $\mathrm{KNO}_{3}$ (кривая 1) и нанокомпозитов на основе $\mathrm{KNO}_{3}$ (кривые 2-4). Из рисунка видно, что общим для всех образцов является наличие дублета - двух пиков с разной интенсивностью с температурами максимумов $T_{\text {о1 }}$ и $T_{\text {o2 }}$, различающиеся на $2-5 \mathrm{~K}$. Природа более высокотемпературного пика в дублете связана с
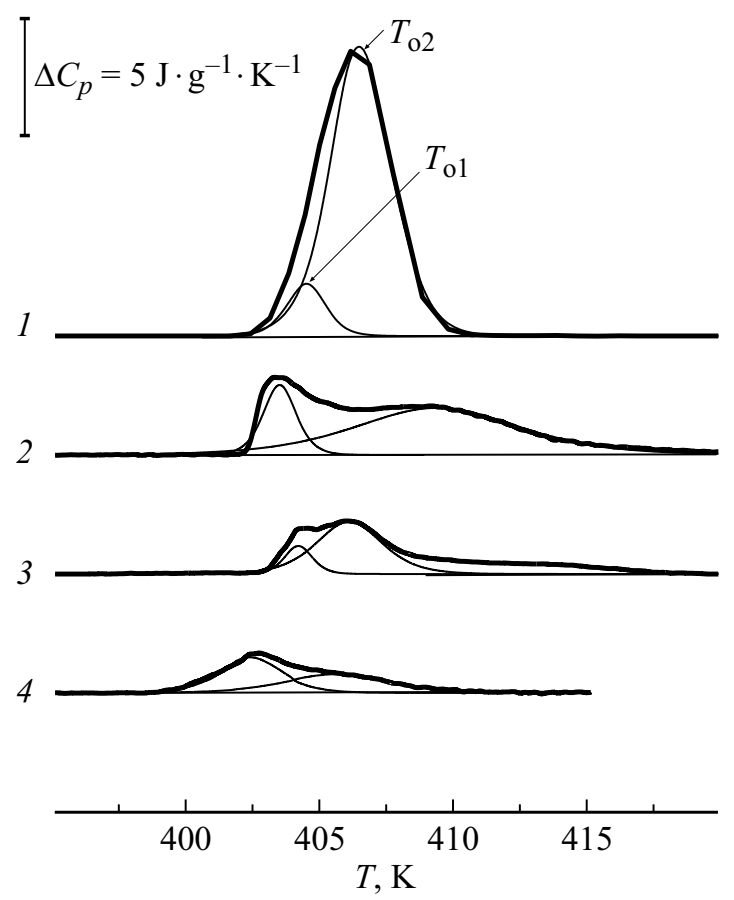

Рис. 4. Кривые ДСК, полученные при нагревании образцов № 1-4 (жирные линии 1-4 соответственно). Тонкие линии - результат расчета теплоемкости по соотношению (1). фазовым переходом в конечную $\beta$-фазу. То есть пик с температурой $T_{\mathrm{o} 2}$ связан с фазовым переходом в объеме кристаллов $\mathrm{KNO}_{3}$. Поскольку имеются данные [13], которые указывают на наличие в нанокомпозите при нагревании сегнетоэлектрической фазы, весьма вероятно, что низкотемпературный пик относится к переходу сегнетоэлектрической $\gamma$-фазы в тригональную кальцитоподобную структуру $\beta$-фазы. Из этого необходимо следует, что во всем температурном интервале от комнатной температуры до $T_{\max } 1$ существует двухфазная структура, состоящая из $\gamma$ - и $\alpha$-фазы. Эта двухфазная структура при уменьшении размера пор в композите будет, по-видимому, смещаться в сторону увеличения доли сегнетоэлектрической фазы, так как замечено тем большее уширение температурной области существования сегнетоэлектрической фазы по сравнению с поликристаллическим $\mathrm{KNO}_{3}$, чем меньше размер пор [2]. На это указывает и наблюдаемое в данной работе существенное увеличение энтальпии в нанокомпозите в сравнении с энтальпией поликристаллического $\mathrm{KNO}_{3}$ для низкотемпературного пика, которое можно представить в виде зависимости относительной доли энтальпии низкотемпературного пика в дублете от размера поры $q_{\mathrm{o} 1} /\left(q_{\mathrm{o} 1}+q_{\mathrm{o} 2}\right)=F(R)$. При этом уменьшение $R$ сопровождается ростом $F(R)$.

Подобная зависимость наблюдается при сравнении объемных долей приповерхностного слоя с объемом частицы при вариации размеров частиц. Действительно, для таких частиц доля атомов, находящихся в объеме $V_{1}$ тонкого поверхностного слоя, в сравнении с объемом микрочастиц $V_{\Sigma}=V_{1}+V_{2}$, где $V_{2}$ - объем сердцевины микрочастицы, заметно возрастает. Объемную долю поверхностного слоя можно оценить по формуле

$$
V_{1} /\left(V_{1}+V_{2}\right)=1-[1-b / R]^{3},
$$

где $b$ - толщина поверхностного слоя, а $R-$ характерный размер частицы. Если принять, что энтальпии переходов в поверхностном слое и объеме микрочастицы равны, то соотношение (4) приобретает вид

$$
F(R)=q_{\mathrm{o} 1} /\left(q_{\mathrm{o} 1}+q_{\mathrm{o} 2}\right)=1-[1-b / R]^{3} .
$$

На рис. 5, помимо экспериментальных точек, представлена в виде кривой зависимость (5) со значением параметра $b=3 \AA$. Видно, что экспериментальные точки удовлетворительно ложатся на эту зависимость. При этом с уменьшением размера зерна объемная доля поверхностного слоя увеличивается. При значениях $R=80-90 \AA$ зависимость $F(R)$ пересекает уровень, отвечающий значениям $R$ для поликристаллического $\mathrm{KNO}_{3}$.

Можно предположить, что в исходном поликристаллическом $\mathrm{KNO}_{3}$ также существует структура, состоящая из наноразмерных $(R=80-90 \AA)$ кристаллов, разделенных неупорядоченными межкристаллическими областями, либо структура, состоящая из т.н. областей когерентного рассеяния (ОКР), разделенных более неупо- 


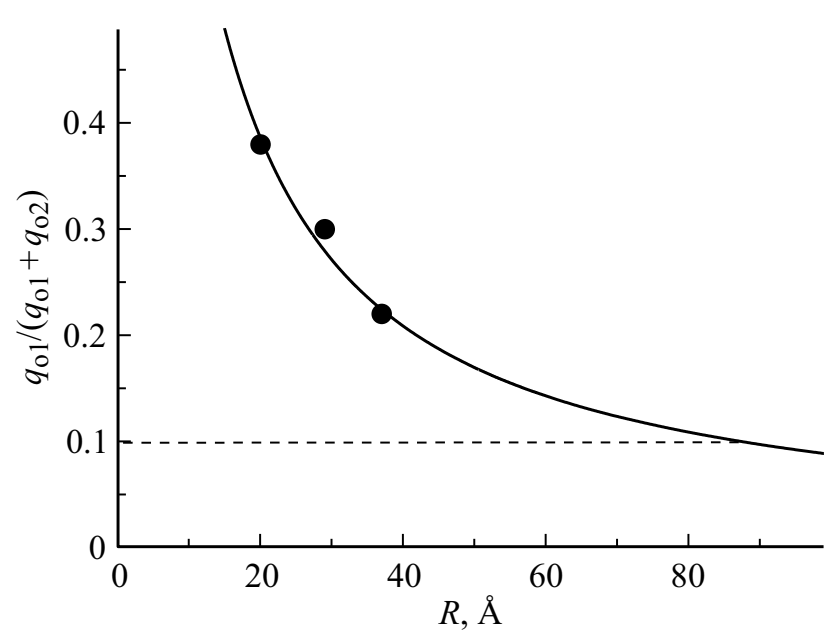

Рис. 5. Зависимость относительной доли энтальпии низкотемпературного пика в дублете от размера поры.

рядоченными поверхностями. Для подтверждения высказанного предположения необходимы дополнительные рентгеновские исследования.

В настоящей работе не выяснен вопрос о типе решетки в поверхностном слое. Известно, что в поверхностном слое малых кристаллических образований возникают сильные искажения кристаллической решетки и даже может происходить смена типа решетки. Если предположить, что тип решетки в поверхностном слое соответствует типу решетки III, которая наблюдается при охлаждении, то нитрат калия в ограниченной геометрии в диапазоне размеров кристаллитов менее 5-10 А будет весь обладать свойствами сегнетоэлектрика. В случае подтверждения изложенных в данной работе результатов можно получить материал с сегнетоэлектрическими свойствами в регулируемом в зависимости от размера нанопор диапазоне.

\section{Конфликт интересов}

Авторы заявляют, что у них нет конфликта интересов.

\section{Список литературы}

[1] С.В. Барышников, Е.В. Чарная, А.Ю. Милинский, Ю.В. Патрушев. ФТТ 55, 2439 (2013).

[2] Ferroelectrics - Material Aspect. / Ed. M. Lallart.-Pablisher. In Tech (2011). 518 p.

[3] J.S. Beck, J.C. Vartuli, W.J. Roth., J. Am. Chem. Soc. 114, 10834 (1992).

[4] Ф. Иона, Д. Ширане. Сегнетоэлекрические кристаллы. Мир, М. (1965). 555 с.

[5] Г.А. Смоленский, В.А. Боков, В.А. Исупов, Н.Н. Крайник, Р.Е. Пасынков, М.С. Шур. Сегнетоэлектрики и антисегнетоэлектрики. Наука, М. (1971). 476 с.

[6] V.V. Deshpande, M.D. Karkhanavala, U.R.K. Rao. J. Thermal Anal. Calorimetry 6, 613 (1974)

[7] K. Illers. Eur. Polym. J. 10, 911 (1974).
[8] V.A. Bershtein, V.M. Egorov. Differential Scanning Calorimetry of Polymers: Physics, Chemistry, Analysis, Technology. Ellis Horwood, N. Y. (1994). 253 p.

[9] М. Фишер. Природа критического состояния. Мир, M. (1973). $260 \mathrm{c}$.

[10] Г.А. Малыгин. УФН 171, 187 (2001).

[11] В. Кенциг. Сегнетоэлектрики и антисегнетоэлектрики. ИЛ, М. (1960). 324 с.

[12] Г.А. Малыгин. ФТТ 43, 1911 (2001).

[13] С.В. Барышников, Е.В. Чарная, А.Ю. Милинский, Ю.А. Шацкая, D. Michel. ФТT 54, 594 (2012).

Редактор К.В. Емцев 